\title{
Implementation and Demonstration of Grid Frequency Support by V2G Enabled Electric Vehicle
}

\author{
Martinenas, Sergejus; Marinelli, Mattia; Andersen, Peter Bach; Træholt, Chresten
}

Published in:

Proceedings of the 49th International Universities Power Engineering Conference (UPEC), 2014

Link to article, DOI:

10.1109/UPEC.2014.6934760

Publication date:

2014

Link back to DTU Orbit

Citation (APA):

Martinenas, S., Marinelli, M., Andersen, P. B., \& Træholt, C. (2014). Implementation and Demonstration of Grid Frequency Support by V2G Enabled Electric Vehicle. In Proceedings of the 49th International Universities Power Engineering Conference (UPEC), 2014 (pp. 1-6). IEEE. https://doi.org/10.1109/UPEC.2014.6934760

\section{General rights}

Copyright and moral rights for the publications made accessible in the public portal are retained by the authors and/or other copyright owners and it is a condition of accessing publications that users recognise and abide by the legal requirements associated with these rights.

- Users may download and print one copy of any publication from the public portal for the purpose of private study or research.

- You may not further distribute the material or use it for any profit-making activity or commercial gain

- You may freely distribute the URL identifying the publication in the public portal 


\title{
Implementation and Demonstration of Grid Frequency Support by V2G Enabled Electric Vehicle
}

\author{
Sergejus Martinenas Mattia Marinelli, Peter Bach Andersen and Chresten Træholt \\ Center for Electric Power and Energy, DTU - Technical University of Denmark \\ Contact Person: Sergejus Martinenas, smar@elektro.dtu.dk
}

\begin{abstract}
Safe operation of the electric power system relies on conventional power stations. In addition to providing electrical energy to the network, some power stations also provide a number of ancillary services for the grid stability. These services could potentially be provided by the growing number of electric vehicles - faster and with better precision, using Vehicle-to-Grid technology. This paper explores the implementation of a system that demonstrates the use of the electric vehicles for providing frequency regulation in the Danish power grid. The system is tested with the use case of Primary Frequency Regulation. The service is implemented following the technical conditions for ancillary services in the Danish grid. The real life system is developed using web-centric communication technologies between the components. Communication and control functions of the system are validated through experiments. The response of the system is studied in terms of latency, precision and stability.

Keywords - Vehicle-to-Grid, Smart Grid, Frequency Control, Electric Vehicles, Information and Communication Technology
\end{abstract}

\section{INTRODUCTION}

Power system frequency stability relies on the balance between power generation and consumption. Today, most of the power generation in the grid is provided by conventional power plants. Sudden generation losses and unexpected load increases require timely actions by the remaining generating units or by controllable loads in order to restore the power balance. Additionally, conventional generating units are displaced by renewable sources, such as wind and photovoltaic, which are not required to provide the same level of ancillary services, like the frequency regulation [1]. This leads to an increase of regulating power obligation among the remaining controllable resources.

Growing number of Electric Vehicles (EVs) is typically viewed as additional load on the grid from system operators perspective [2]. However, EVs are also one of the imminent candidates for providing grid regulation services. Especially, because most of the time (typically about 90\%) they are plugged-in to a charging post and can provide fast regulating power in both directions. The ability of the vehicle to provide power back to the grid is called Vehicle-to-Grid (V2G) [3]. A noticeable amount of literature has already been written

This work is supported by the Danish Research Project "NIKOLA Intelligent Electric Vehicle Integration"- under ForskEL kontrakt nr. 20131-12088. More information (www.nikolaproject.info). supporting the use of EVs with V2G capability for ancillary services [4], [5]. However, multiple technical challenges arise: the limited power and energy capacity of each individual unit and the need of having simple and effective measurement and control capabilities [6]. These problems are solved by aggregating a large number of V2G enabled vehicles [7] and connecting the aggregator to the high quality measurement device.

This work focuses on the system design and analysis of the issues that may arise when dealing with practical implementation of frequency response such as: communication latency, power and frequency measurements inaccuracies. Therefore, the implementation of the system and communication to the electric vehicle will be presented in detail.

The paper is organized as follows: Section II provides the background on grid frequency regulation services, with the example of Danish grid. Section III gives an overview on system implementation with focus on the communication implemented using a modern web-centric approach. Section IV shows the results of a frequency measurement and frequency regulation experiments. Section V presents discussion of the results.

\section{Frequency Regulation Services}

Generally, the grid frequency regulation reserves are divided into the three categories: primary, secondary and tertiary. The Danish power grid is located in two separate synchronous regions: DK1 and DK2. Jylland (continental part of Denmark) and island of Fyn are in DK1 region connected to the rest of Europe. The islands of Sjælland and Bornholm are in DK2 connected to most of Scandinavia. In each region, there are multiple levels of reserves providing frequency regulation services [8]. DK1 region has following frequency regulation reserves:

- Primary Reserve - regulation is restoring balance between production and consumption, stabilizing the frequency close to $50 \mathrm{~Hz}$. The regulation is automatic, power output of the reserve is controlled according to the frequency deviation, with the small permitted deadband.

- Secondary Reserve - regulation is enabled during major operational disturbance. It is used to indirectly restore frequency to $50.00 \mathrm{~Hz}$ followed by stabilization by primary reserve. It also serves the purpose of releasing the 
activated primary reserve. The regulation is automatic and controlled by a signal from Energinet.dk.

- Manual Reserve - manual power regulation used to restore system balance, acting as a tertiary reserve. It is activated manually from Energinet.dk's Control Center.

DK2 region also has corresponding reserves:

- Normal Operation Reserve - ensures that production and consumption equilibrium is restored. The regulation is automatic and responding to frequency deviation, without deadband.

- Disturbance Reserve - a fast reserve, activated in the event of major system disturbances. It is started automatically in the event of sudden frequency drop under $49.9 \mathrm{~Hz}$ and remains active until frequency is restored or manual reserve takes over.

- Manual Reserve - manual power regulation used to restore system balance, acting as a tertiary reserve, manually activated from Energinet.dk's Control Center.

In this paper the primary reserve from DK1 is implemented and tested. It is chosen for experiments, as the reserve should directly respond to grid frequency deviations, it has a small optional deadband and requires a quick response time.

\section{System ARChitecture}

To implement the frequency regulation service the reserve has to have three main components: a controllable energy resource connected to the grid, fast and precise grid frequency measurements and a controller with appropriate control algorithm.

\section{A. System Design}

The system developed in this project, is split into three layers, shown in Fig. 1: power flow, data acquisition and intelligence.
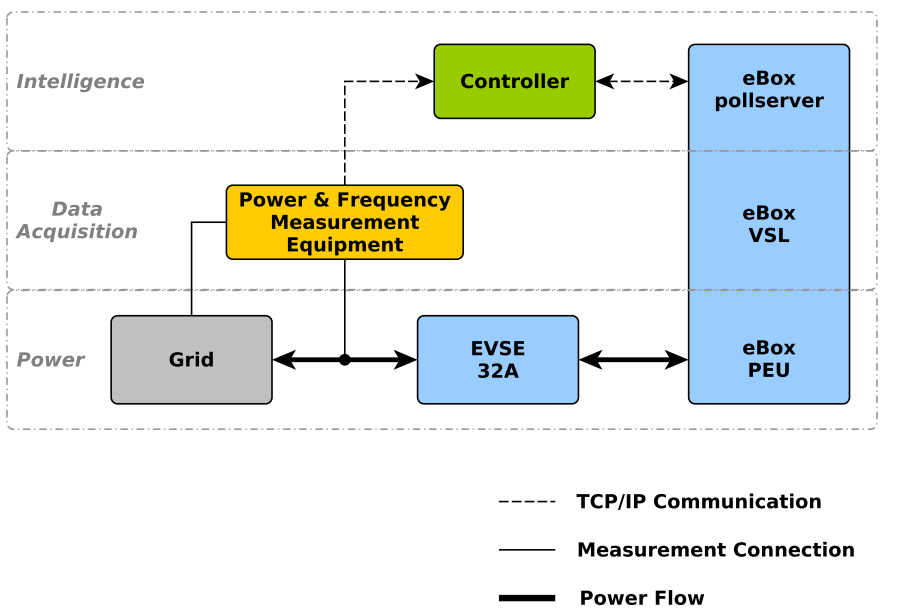

Fig. 1. System design for the frequency regulation service

The power layer consists of all the devices that contain power electronics or are used to generate or transmit electrical energy, e.g. EV battery, inverter, charging cables, breakers, etc. Data acquisition layer consists of the embedded and external measurement devices and their interfaces. The intelligence layer contains all the computers running higher level communication and control algorithms e.g. EV computer and controller server.

As shown in Fig. 1 the testing platform was build with following components:

- EV - DTU eBox with 35kWh battery and power electronics unit (PEU) unit capable of single phase bidirectional power transfer up to $20 \mathrm{~kW}$. It is controlled by the EV computer that interfaces with the PEU using build-in vehicle smart link (VSL) [9].

- VSL - an embedded computing interface, used for communication to eBox PEU and is located next to it. Additionally the VSL is responsible for handling IEC 61851 Control Pilot signal communication, when the vehicle is plugged in to the EVSE.

- EV Computer - build-in EV computer running the eBox pollserver application. It is connected to the network using wireless interface.

- eBox Pollserver - vehicle monitoring/control server with RESTful application programming interface (API). It is implemented on the vehicle computer and is used for remote and local control.

- EVSE - grid connected, 32A, three phase, custom charging spot with charging cable.

- Frequency Measurement Device - G4500 Power Analyzer from ELSPEC, highly accurate grid analysis unit, primarily used for grid frequency measurement, also connected to communication network.

- Frequency Regulation Controller - running on external computer, connected to network, performing following functions: reading data from measurement devices and dispatching control signals to the EV.

All of the system components have communication interfaces for control and data exchange.

\section{B. Electric Vehicle Communication}

The system layers: grid connected EV, frequency measurement device and controller, were distributed in the different places of the SYSLAB ${ }^{1}$ laboratory and connected to the common communication network. The controller and measurement device are using wired network connection, the EV computer is connected using wireless communication. These physical layers are chosen for its robustness, flexibility and representation of the real field testing conditions. The full communication path from controller to the PEU is shown in Fig. 2.

The EV computer is running a pollserver application, which uses the connection to the VSL board to update the data values and control parameters. The pollserver receives and processes the requests from the controller into updated EV control commands or returns measurement data. The VSL is running an

${ }^{1}$ SYSLAB is laboratory for intelligent distributed power systems at Technical University of Denmark, part of PowerLabDK (www.powerlab.dk). 


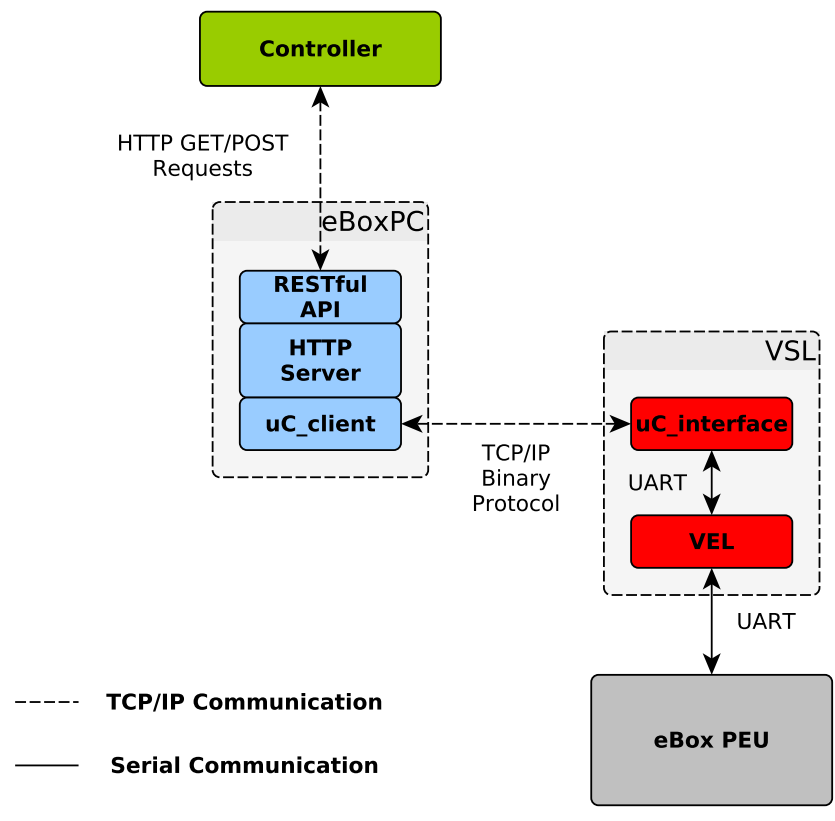

Fig. 2. Control message path from controller to the PEU

interface application, that sends those commands to the PEU unit. The higher level communication is implemented based on Hyper Text Transfer Protocol (HTTP). On the embedded side of the system (on the VSL and below) communication changes to serial UART, which is a common component used in industry for such applications.

As shown in the Fig. 2 high level communication has been implemented for communication with the EV using RESTful architecture. REpresentational State Transfer (REST) is a software architectural style rather than a communication protocol. It has first been described in the $\mathrm{PhD}$ thesis of R.Fielding [10]. REST description consist of a set of architectural constraints applied to components and data elements, within a system. It ignores the implementation details of the component and protocol syntax, focusing on the component roles, their interaction and interpretation of significant data elements. RESTful architecture have been used to map the IEC 61850 communication standard to web services [11]. The communication protocol is kept as simple and clear as possible to ease later expansion and higher level application such as IEC 15118. The structure of RESTful Unique Resource Identifier (URI) request, designed for this work, is shown in Fig. 3.

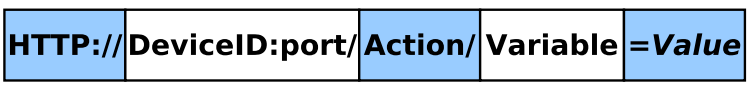

Fig. 3. RESTful URI request structure

Following is the RESTful API communication example: To read the measurements or parameters of the EV, an HTTP GET request is sent to the URI:

http://ebox:8080/getData/measurements
The response received is formatted in JavaScript Object Notation (JSON):

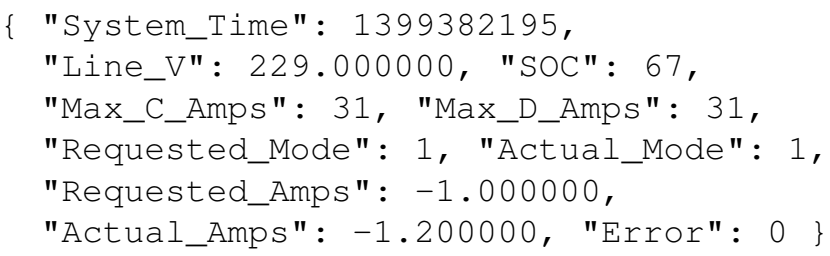

For setting new control parameters, such as charging current or operation mode, a HTTP POST or PUT request is sent. For example, to update the charging current to -16A (negative values indicate charging, positive values indicate V2G mode, i.e. discharging into the grid), the HTTP POST request is sent to the following URI:

http://ebox: $8080 /$ setData/Requested_Amps $=-16$

To update the operation mode from charging (mode 1) to V2G (mode 2) following HTTP POST request is sent:

http: //ebox: $8080 /$ setData/Requested_Mode=2

This communication API is used by the controller application to read the data and control the EV.

\section{Primary Frequency Regulation Controller}

The frequency regulation controller is running with the following algorithm:

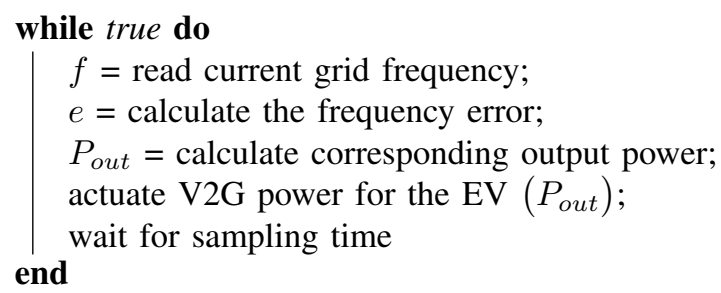

\section{Algorithm 1: Frequency Droop Control}

The Primary Frequency Regulation (PFR) controller was designed by following the technical specification of tender conditions for ancillary services in Danish power grid, see [8].

First the grid frequency is measured and the deviation from nominal frequency is calculated:

$$
e=f_{\text {nom }}-f_{m}
$$

Here the $e$ is the deviation i.e. the difference between nominal and measured frequency values. $f_{\text {nom }}$ is a nominal grid frequency, in Europe it is $50.00 \mathrm{~Hz}$. The $f_{m}$ is the momentarily measured grid frequency, with $10 \mathrm{mHz}$ accuracy. Droop controller output is calculated as shown in (2).

$$
P_{\text {out }}= \begin{cases}0 & ,|e|<\Delta f_{d b} \\ P_{\max } \cdot e \cdot \frac{100}{d_{p c t}} & , \Delta f_{\max }>|e|>\Delta f_{d b} \\ P_{\max } & ,|e|>\Delta f_{\text {max }}\end{cases}
$$


Here $P_{\text {out }}$ is output power of the vehicle to the grid. $P_{\max }$ is the maximum output power of the vehicle, for a $32 A$ charging spot at one phase it is $7 k W . \Delta f_{d b}$ is a deadband used, in this case $20 \mathrm{mHz} . \Delta f_{\max }$ is a maximum frequency deviation, at which the maximum power should be output to the grid, in this case it is $200 \mathrm{mHz}$. The resulting droop control curve is shown in Fig. 4.

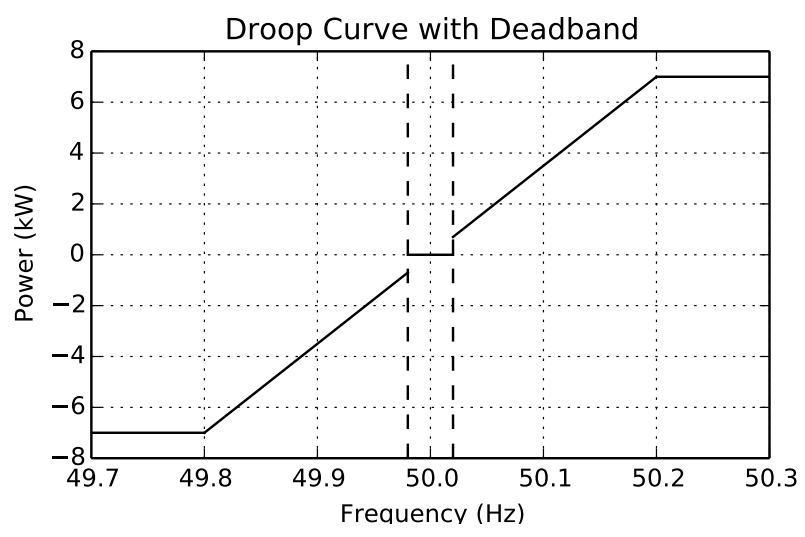

Fig. 4. Droop curve used for frequency regulation experiments

\section{EXPERIMENTAL RESULTS}

\section{A. System Frequency Measurements}

As specified in the ancillary service conditions, each frequency reserve must measure the grid frequency with accuracy better than $10 \mathrm{mHz}$. Therefore a precise grid power analyzer was installed at the permanent grid connection in SYSLAB. The data from the measurement equipment was recorded from April 2nd to April 30th. All frequency values are plotted in Fig. 5.

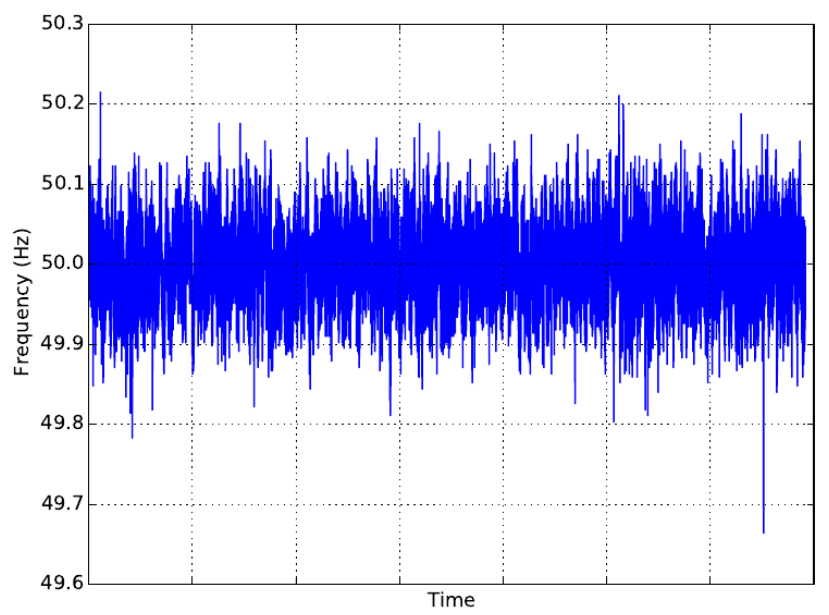

Fig. 5. Frequency data values plot for April 2 to April 30

Collected frequency data is analyzed with results shown in Table I.
TABLE I

FREQUENCY DATA CHARACTERISTICS FOR APRIL

\begin{tabular}{|c|c|c|c|}
\hline Min Value & Max Value & Mean & Standard Deviation \\
\hline $49.664 \mathrm{~Hz}$ & $50.215 \mathrm{~Hz}$ & $50.001 \mathrm{~Hz}$ & $0.0425 \mathrm{~Hz}$ \\
\hline
\end{tabular}

This analysis and frequency distribution in Fig. 6 shows that frequency is highly symmetric over longer periods of time, thus the amount of energy supplied by the EVs for up regulation should equal to the energy consumed by the vehicles for down regulation. However, the experimental example will show, that the energy taken and received back to the battery is not equal, due to efficiency of the charger, at lower current values being around $80 \%$, and only up to $92 \%$ at higher charging power. This will result in significant drop of the battery SOC, on the longer symmetric regulation periods.

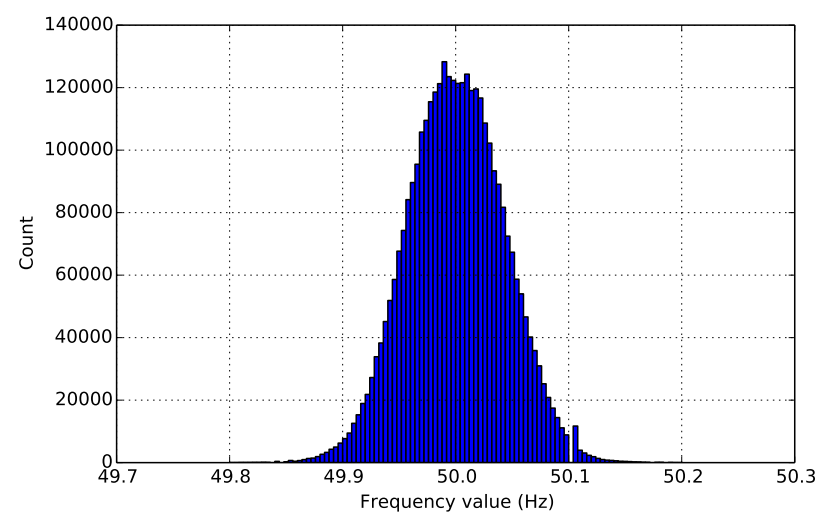

Fig. 6. Frequency data value distribution from April 2 to April 30

In the histogram in Fig. 6, the bin width is $4 \mathrm{mHz}$. Most of the frequency values $-99.99 \%$, are between $49.8-50.2 \mathrm{~Hz}$. The symmetrical spikes at bins $49.988-49.992 \mathrm{~Hz}$ and $50.008-50.012 \mathrm{~Hz}$ are corresponding to a metering-related deadband of some primary frequency regulation controllers. This deadband could occur because of the frequency measurement devices used to activate the controllers. The ancillary service conditions states, that the accuracy and sensitivity of the frequency meters must be better than $\pm 10 \mathrm{mHz}$ [8]. Therefore, it may happen that some power units, especially small ones which may be equipped with less sensitive metering devices, are not activated while the frequency is within $50.00 \mathrm{~Hz} \pm 10 \mathrm{mHz}$.

\section{B. Frequency Regulation}

The results of the frequency regulation: each plot shows the grid frequency values, state of charge (SOC) of the EV battery and the $\mathrm{V} 2 \mathrm{G}$ current from the EV to the grid. The positive current indicates that the vehicle is providing power to the grid and with the negative current values, EV is consuming power i.e. charging. 
In the first experiment, the vehicle was plugged in overnight providing primary frequency regulation for 6 hours. Experiment result is shown in Fig. 7.

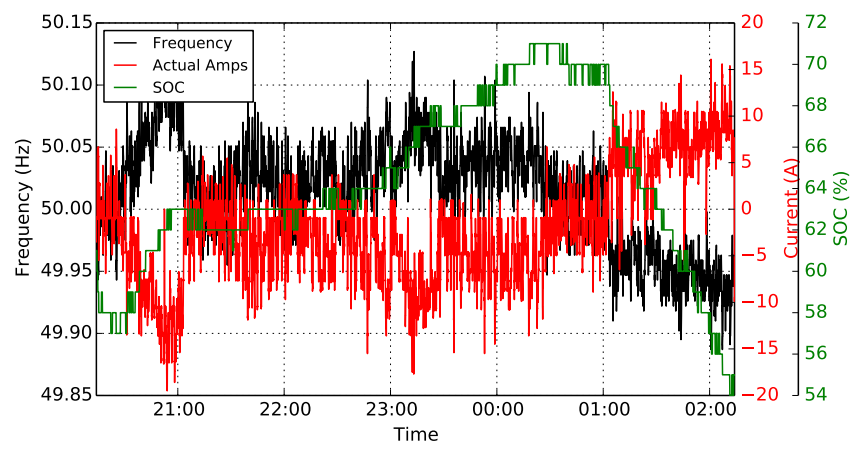

Fig. 7. Frequency regulation results for 6 night hours

Here one can observe that the frequency (marked in black) is above normal most of the night, thus the vehicle is charging at up to $20 A$ (marked in red). The SOC of the vehicle (marked in green) is also balancing above the starting value, at the peak reaching $71 \%$. However, at $3 \mathrm{am}$ the frequency drops below the nominal value and the vehicle switches to the $\mathrm{V} 2 \mathrm{G}$ mode. At the end of the experiment the EV has $54 \%$ SOC.

A longer experiment was also performed with frequency regulation being active for 22 hours. The results are shown in Fig. 8.

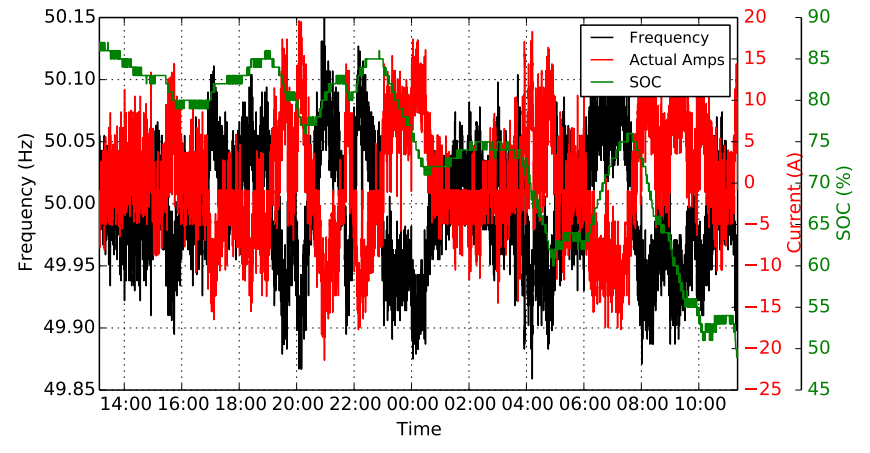

Fig. 8. Frequency regulation results for 22 hours

Here the average frequency value for the test period is $49.997 \mathrm{~Hz}$. This indicates that the frequency is almost symmetric, with a slight bias towards the value below nominal. The SOC of the vehicle changes from $85 \%$ at the start to around $53 \%$ at the end of the test. The change can be explained by slight bias of the frequency and mainly by the V2G converter efficiency. The measured efficiency of vehicle PEU in V2G mode at these low current values is around 0.8 , so the roundtrip efficiency results in around 0.64 . This corresponds to the ratio of the end to start value of battery SOC in the example from Fig. 8.

Further, the close up from the 22 hour frequency regulation process is shown in Fig. 9.

In this plot the delay between the frequency measurement and response by the vehicle can be appreciated. Generally, the

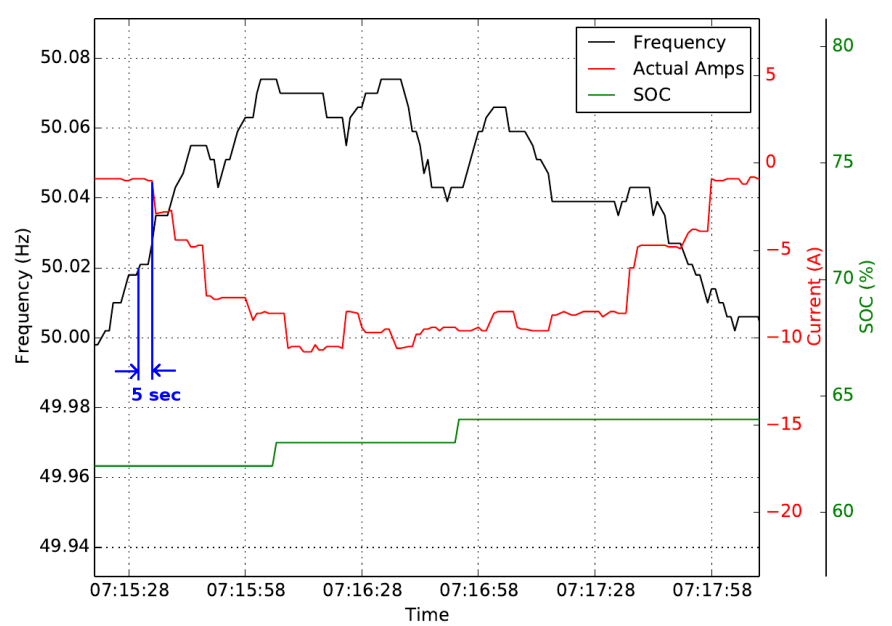

Fig. 9. Frequency regulation results close up with response delay

eBox is able to respond to the frequency change in 5 to 6 seconds on average. This delay consists of approximately: 1 second delay for frequency sampling time and control action in the controller, 1 second delay for communication and control parameter update in the pollserver, 3 to 4 seconds for PEU ramping time and 1 second delay for response data sampling in the measurement device.

All tests were performed in SYSLAB - a DTU research facility for intelligent, active and distributed power systems. SYSLAB is located at DTU Ris $\varnothing$ campus and is a part of the PowerLabDK experimental facilities.

\section{Discussion}

The primary frequency regulation based on an electric vehicle proved successful in operation. Web-centric communication protocols introduce application flexibility, platform independence and significantly speed up controller implementation. The response of the vehicle to the detected frequency deviation is much faster than the specified technical conditions for regulation: $5-6 \mathrm{~s}$ versus $15 \mathrm{~s}$. This experimentally proves that bi-directional power exchange with electric vehicles are fast enough for providing this kind of grid support [12]. Even faster response times can be achieved by increasing data sampling and control parameter update frequency, however most of the delay is due to the slow ramping rate of the PEU unit. Overall the response delay could be reduced to less than half - around 2-3s. Several improvements are proposed for better EV integration for grid frequency support: the PEU efficiency should be improved to minimize the roundtrip energy losses; the battery degradation should be taken into account and studied in detail as different studies suggest contradictory results [13], [14]. However, it has been proven in multiple studies, that providing grid services using EVs is economically beneficial [15], [16]. Finally, this work has shown, that reallife application of the EV for grid frequency support is feasible and modern communication technologies help the intelligent integration of the EV for providing grid services [17]. 


\section{REFERENCES}

[1] M. Marinelli, S. Massucco, A. Mansoldo, and M. Norton, "Analysis of inertial response and primary power-frequency control provision by doubly fed induction generator wind turbines in a small power system,' Proceedings of the 17th Power Systems Computation Conference, Stockholm, 22-26 Aug. 2011, 2011.

[2] K. Clement-Nyns, E. Haesen, and J. Driesen, "The impact of charging plug-in hybrid electric vehicles on a residential distribution grid," Power Systems, IEEE Transactions on, vol. 25, no. 1, pp. 371-380, Feb 2010.

[3] W. Kempton and J. Tomic, "Vehicle-to-grid power implementation: From stabilizing the grid to supporting large-scale renewable energy," Journal of Power Sources, vol. 144, no. 1, pp. 280 - 294, 2005.

[4] H. Lund and W. Kempton, "Integration of renewable energy into the transport and electricity sectors through V2G," Energy Policy, vol. 36, no. 9 , pp. 3578-3587, 2008.

[5] J. Pillai, B. Bak-Jensen, and P. Thogersen, "Electric vehicles to support large wind power penetration in future danish power systems," in Vehicle Power and Propulsion Conference (VPPC), Seoul, 09-12 Oct. 2012 IEEE, Oct 2012, pp. 1475-1479.

[6] F. Marra, D. Sacchetti, A. Pedersen, P. Andersen, C. Træholt, and E. Larsen, "Implementation of an electric vehicle test bed controlled by a virtual power plant for contributing to regulating power reserves," Power and Energy Society General Meeting, 2012 IEEE, no. 081216, pp. 1-7, 2012.

[7] S. Han, S. H. Han, and K. Sezaki, "Design of an optimal aggregator for vehicle-to-grid regulation service," Innovative Smart Grid Technologies (ISGT), 2010, 2010.

[8] "Ancillary services to be delivered in Denmark Tender conditions," Tech. Rep. October, 2012.

[9] University of Delaware, "The Grid-Integrated Electric Vehicle," Tech. Rep.

[10] R. T. Fielding, "Architectural styles and the design of network-based software architectures," Ph.D. dissertation, 2000, AAI9980887.

[11] A. Pedersen, E. Hauksson, P. Andersen, B. Poulsen, C. Træholt, and D. Gantenbein, "Facilitating a Generic Communication Interface to Distributed Energy Resources: Mapping IEC 61850 to RESTful Services," in Smart Grid Communications (SmartGridComm), 2010 First IEEE International Conference on, Maryland, 17-20 Oct 2010, pp. 61-66.

[12] J. R. Pillai and B. Bak-Jensen, "Vehicle-to-grid systems for frequency regulation in an Islanded Danish distribution network," 2010 IEEE Vehicle Power and Propulsion Conference, pp. 1-6, 2010.

[13] S. Peterson, J. Apt, and J. Whitacre, "Lithium-ion battery cell degradation resulting from realistic vehicle and vehicle-to-grid utilization," Journal of Power Sources, vol. 195, no. 8, pp. 2385-2392, Apr. 2010.

[14] S. You, J. Hu, A. Pedersen, P. Andersen, C. Rasmussen, and S.-T. Cha, "Numerical comparison of optimal charging schemes for electric vehicles," Power and Energy Society General Meeting, 2012 IEEE, pp. $1-6,2012$.

[15] W. Kempton and J. Tomić, "Vehicle-to-grid power fundamentals: calculating capacity and net revenue," Journal of Power Sources, vol. 144, no. 1, pp. 268-279, Jun. 2005.

[16] S. Han and K. Sezaki, "Economic assessment on V2G frequency regulation regarding the battery degradation," Innovative Smart Grid Technologies (ISGT), 2012 IEEE PES, pp. 1-6, 2012.

[17] A. Pedersen, P. Andersen, J. Skov Johansen, D. Rua, J. Ruela, and J. A. Pecas Lopes, ICT Solutions to Support EV Deployment, ser. Power Electronics and Power Systems. Springer-Verlag, New York, 2013, pp. $107-154$. 